\title{
Retrospective analysis of chest x-ray of patients from eastern Uttar Pradesh presenting in Radiology department of Hind Institute Medical Sciences, Barabanki Uttar Pradesh
}

\author{
Khare R. ${ }^{1}$, Khare A. ${ }^{2 *}$
}

DOI: https://doi.org/10.17511/ijmrr.2019.i02.08

\footnotetext{
${ }^{1}$ Rekha Khare, Associate Professor, Department of Radio-diagnosis, Hind Institute of Medical Sciences, Barabanki, Uttar Pradesh, India.

2* A.K. Khare, Associate Professor, Department of Pharmacology and Honorary Physician, Hind Institute of Medical Sciences, Barabanki, Uttar Pradesh, India.
}

Introduction: Chest Xray is one of the most frequently asked radiological examination in patients with heart or lung problems in general medical practice. There are many studies on correlation of CXR findings and other tools of investigations like CT scan, MRI and laboratory investigations like spirometry and sputum examinations etc. The present study aims at establishing a possible correlation in rural population of Eastern Uttar Pradesh. Methods: We have done a cross sectional study in a period of six months including 2500 patients coming for CXR. Patients were followed up to a point where radiological diagnosis was confirmed or otherwise after other investigations and clinical response. Results: There was fairly good correlation in patients with COPD (73.15\%), tuberculosis (85.8\%), interstitial lung disease $(63.9 \%)$ and congestive heart failure (68.6\%). Conclusion: We conclude that $\mathrm{CXR}$ is tool of reasonable sensitivity however there is sizeable possibility of false positive and negative cases.

Keywords: Persistent cough, Lung conditions, Pneumothorax

Corresponding Author

A.K. Khare, Associate Professor, Department of Pharmacology and Honorary Physician, Hind Institute of Medical Sciences, Barabanki, Uttar Pradesh, India. Email: akharester@gmail.com
How to Cite this Article

Khare R, Khare AK. Retrospective analysis of chest $x$ ray of patients from eastern Uttar Pradesh presenting in Radiology department of Hind Institute Medical Sciences, Barabanki Uttar Pradesh. Int J Med Res Rev. 2019;7(2):98-103.

Available From

https://ijmrr.medresearch.in/index.php/ijmrr/article/ view/1043

\section{To Browse}

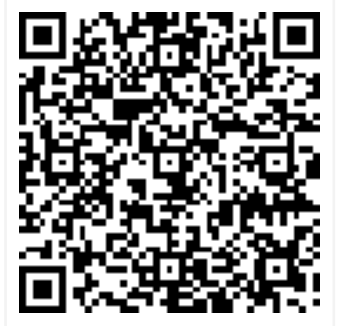

Manuscript Received 2019-04-10

Conflict of Interest No

(c) 2019 by Rekha Khare, A.K. Khare and Published by Siddharth Health Research and Social Welfare Society. This is an Open Access article licensed under a Creative Commons Attribution 4.0 International License https://creativecommons.org/licenses/by/4.0/ unported [CC BY 4.0].

Accepted 2019-04-30

Review Round 2019-04-20

Funding

view Round 2

2019-04-26

Review Round 3

Note 


\section{Introduction}

Chest x-ray uses a very small dose of ionizing radiation to produce pictures of the inside of the chest. It is used to evaluate the lungs, heart and chest wall and may be used to help diagnose shortness of breath, persistent cough, fever, chest pain or injury. It also may be used to help diagnose and monitor treatment for a variety of lung conditions such as pneumonia, emphysema and cancer. Because chest $x$-ray is fast and easy, it is particularly useful in emergency diagnosis and treatment. We therefore planned the present study and publish the results and discuss them in view of other contemporary studies $[1,2,3]$.

We are presenting the pattern of radiological diagnoses of 2500 patients attending the department of radiology for $x$-ray chest in the radiology. The burden of chest disease is huge in our area on account of poor socioeconomic status. Though somedata on major lungproblems like tuberculosis [1] COPD [2,3] and lung mass $[4,5]$ is available but data on different lung diseases is very limited $[6,7]$.

\section{Patients and Methods}

Radiology department of Hind Institute of Medical Sciences, Safedabad is well equipped, withadequate number of trained radiologists and competenttechnical staff.

Study type: A total of 2500 adultpatients (above 18 years) of either sex were included in thiscrosssectional study. Most patients were attending medical out-patient department of Hind Institute or referred by private medical practitioners in rural and urban clinics nearby during the period of November 2017 to April 2018. In most of the cases diagnosis was limited to major findings of the disease for the purpose of analysis.

Further subgroup analyses were not attempted as diagnostic testsneeded to establish completediagnosis could not be done due to logistics of private hospital setting and poor socioeconomic status of the patients. The best possible effort was made to reach a radiological diagnosis, contrast CT scan or HRCT was done wherever it was required and whenever it was possible.

Cases were mainly diagnosed on the basis of radiological signsand in correlation of clinical and lab findings, wherever possible.
Spirometry: Sprirometry was done in Respiratory medicine department by trained technician using Fleisch Pneumotach spirometer which was calibrated daily. (8)

Statistics: Descriptive statistics was used to describe results in terms of percent patient in each group as shown in table and accompanying chart diagram.

Ethics: The study was based on routine clinical work done in the department. No ethical issues were involved.

Only competent staff carried out procedures following prescribed protocol. Patients were treated and followed up by their respective physicians. No surgery or interventional procedure was done in any patient.

\section{Results}

01. Chronic obstructive air way disease is an umbrella term for a group of lung disease with shortness of breath and cough with sputum diagnosed on gold guideline.

Radiologically it is seen as emphysema and chronic bronchitis / bullae and or pneumothorax. Confirmed on HRCT in many cases where there was strong history of tobacco smoking. Later the diagnosis was confirmed on lung function tests which showed FEV1 /FVCreduced by more than 25\%. (Chhabra et al, 2014)[3].

02. Tuberculosis was seen on X-ray chest as heterogenous lesion or pleural effusion or cavitating lesion or miliary mottling with or withoutly mphadenopathy and later confirmed by sputum examinations (3 samples) as per protocol for AAFB [9] and if required culture for mycobacterium tuberculosis was done.

03. Lung mass was seen as a soft mass lesion or atypical pneumonia with or without pleural effusion with or without rib erosion or single or multiple nodular mass lesion with known malignancy elsewhere, cases were later sent for PETCT or fine needle aspiration cytology of mass or any accessible lymph node.owever, some patients (25) didn't come back for feedback and all of them had poor prognosis.

04. Radiological signs for Interstitial lung disease were not very specific but cases were strongly suspected in clinico-radiological correlation where diffuse infiltrative opacification in the periphery of the lung or ground glass changes like that of pneumonitis are found. 
Further confirmation was done on HRCT scan of thorax. Due to financial constraints and other logistic problems bronchoscopy and endobronchial biopsy or bronchial lavage could not be done.

Most cases were quite advanced with poor prognosis.

05. Pleural disease / effusion or empyema were diagnosed on chest xray, later confirmed on ultrasound guided pleural fluid aspiration, cytology and HRCT
06. Infective problems /pneumonia were diagnosed in correlation with clinical picture.

High grade fever with cough with expectoration with leukocytosis in CBC.

Radiological sign of alveolar opacity / segmental homogenous opacity with air bronchogram was noted.

CBC and sputum examination for Gram's staining and sputum culture was done.

Table showing analysis of 2500 Chest X-rays

\section{done in HIMS.}

\begin{tabular}{|c|c|c|c|c|}
\hline S. & $\mathrm{X}$ ray Diagnosis & $\begin{array}{l}\text { Number of patients } \\
\text { diagnosed on XXR }\end{array}$ & $\begin{array}{l}\text { Number of patients diagnosed after lab tests and } \\
\text { therapeutic response }\end{array}$ & $\begin{array}{l}\text { Percentage of total cases } \\
\text { under study }\end{array}$ \\
\hline 1 & Normal & 1428 & & 57.12 \\
\hline 2 & $\begin{array}{l}\text { Chronic obstructive Pulmonary } \\
\text { disease (COPD) }\end{array}$ & 298 & $218(73.15 \%)$ & 11.92 \\
\hline 3 & Tuberculosis & 268 & $230(85.8 \%)$ & 10.72 \\
\hline \multirow[t]{5}{*}{4} & Pleural pathology & 160 & & 6.4 \\
\hline & Pleural effusion & 60 & $37.4 \%$ & 2.4 \\
\hline & Pneumothorax & 16 & $10.0 \%$ & 0.64 \\
\hline & Masses & 04 & $2.5 \%$ & 0.16 \\
\hline & Thickening & 80 & $50.0 \%$ & 3.2 \\
\hline \multirow[t]{2}{*}{5} & Cardiogenic lung congestion & 102 & $68(66.6 \%)$ & 3.52 \\
\hline & Non cardiogenic lung congestion & 14 & - & 0.56 \\
\hline 6 & Interstitial lesions & 86 & $55(63.9 \%)$ & 3.44 \\
\hline 7 & Lung mass including metastasis & 66 & $8(12.2 \%)$ & 2.64 \\
\hline 8 & lymphadenopathy & 44 & $3.08 \%$ & 1.76 \\
\hline
\end{tabular}

\section{Discussion}

Chest X-rays are a common type of examination. A chest $X$-ray is often among the first procedures if doctor suspects a patient may have heart or lung disease. It can also be used to check how a patient is responding to treatment.

Our study is mainly OPD based cross sectional study and descriptive analysis of data.It may not be proper epidemiological survey but still it is important.

Our study shows highest prevalence is of chronic obstructive lung disease, followed byinfective lesion predominantly tuberculosis, interstitial lung disease and then mass lesion.

Chronic obstructive pulmonary disease- Chronic obstructive pulmonary disease (COPD) is a pathologic pulmonary condition characterized by expiratory airflow obstruction due to emphysematous destruction of the lung parenchyma and remodeling of the small airways.
While spirometry is a very useful diagnostic tool for screening large groups of smokers, it cannot readily differentiate the etiologies of COPD and thus has limited utility in characterizing subjects for clinical and investigational purposes. While chest $x$-ray is almost universally available, it lacks sensitivity in detecting both airway disease and mild emphysema.

It is an umbrella term for a group of lung disease with shortness of breath and cough with sputum diagnosed on GOLD guide line.Radiologically it was emphysema and chronic bronchitis/bullae and/ or pneumothorax. Which wasconfirmed on HRCT. In many cases there was strong history of tobacco smoking. Later the diagnosis was confirmed on Lung functions tests showing reduced FEV1/FVC ratio (less than $75 \%$ )

COPD may need to be differentiated from other causes of shortness of breath such as congestive heart failure, pulmonary embolism, pneumonia or pneumothorax. Many people with COPD mistakenly thought to be suffering fromasthma [10]. 
The distinction between asthma and COPD is made on the basis of the symptoms, History ofsmoking and whether airflow limitation is reversible with bronchodilators at spirometry [11]. In our study, 298 patients were diagnosed to have COPD on CXR but after spirometry only $218(73.15 \%)$ patients were found to have FEV1/ FVC ratio below $75 \%$ of predicted value.

This discrepancy shows overdiagnosis on CXR. Additionally, CXR did not correctly categorize COPD in mild, moderate or severe. In a recent study (2016) of Pudney and Moharty [12] One third of patients with positive $x$-ray reports did not have COPD on spirometry. Chugh et al [13] have found in a contemporary study that $\mathrm{CT}$ scan was a better method in diagnosis of emphysema seen on CXR as it correlated well with physiological parameters.

Keeping people away from starting to smoke is a key aspect of preventive policies of governments, public health agencies, and ant-smoking campaigns that can reduce smoking rates by discouraging people from starting and encouraging people to stop smoking [14]. Banning smoking in public areas and places of work are important measures to decrease exposure to secondhand smoke, and while many places have instituted bans, more are recommended [15] Though community based data regarding COPD is scantier the different prevalence in different parts could explained by the varied tobacco smoking habit in different part of country

Tuberculosis: In present study, infective problems including tuberculosis consist of the $10.75 \%$ of total number of cases. Tuberculosis- Experts believe that one third of the world population is infected with $M$. tuberculosis [5]. New infections occur at a rate of one per second. In 2007, about 13.7 million chronic cases were active globally [6]. In 2010, about 8.8 million new cases developed and nearly 1.5 million people died of disease, most of them in developing countries [7]. The number of tuberculosis cases has been decreasing since 2006, and new cases have decreased since 2002 [7].

In present study, diagnosis wasmade in 268 (10.75\% of total) cases of TB by radiological signs and clinical examination. However, sputum examination for AAFB and /or sputum culture diagnosed only 190(70.89) cases. These results of sputum examination for AAFB included the retrospective analysis using routine recordsfrom lab of respiratory medicine and therefore are not absolutely unbiased (9).
In some cases, therapeutic response to ATT after one month was used as criteria to establish diagnosis. CXR has historically been used as a first tool to diagnose TB, especially pulmonary TB and thus a valuable tool to identify TB as differential diagnosis. However, CXR has poor specificity.

Moreover, there is significant difference in intraobserver and interobserver variation in reading CXR.This studysuggests that TB, an infectious disease, should be diagnosed by demonstration of M. tuberculosis on culture or A FB on smear microscopy [16]. WHO has classified TB into microbiologically proved TB and clinical TB (based on clinical diagnosis and CXR and therapeutic response) and has clearly emphasized that diagnosis of TB on CXR alone can lead to problem of underdiagnosis as well as over diagnosis [17].

In one study completed in Nairobi, Kenya including 1389 patients found the sensitivity/ specificity of the CXR score "TB" among smear-negative suspects was $80 \% / 67 \%$ [18] TB may mimic other diseases on $\mathrm{x}$-rays, and non TB conditions may look like TB.

Therefore, we suggest that all persons with chest radiographic findings suggestive of TB should have sputum specimens submitted for microbiological examination. CT scan is not cost effective and should be reserved in selected cases.

Interstitial lung disease:In present study interstitial lung disease was seen in 86 (3.4\%) cases.The patterns of lung damage associated with various types of interstitial lung disease are often identifiable on chest $x$-rays. Chest $x$-rays may also be used to track the progression of interstitial lung disease.

Chest radiography is usually the first test to detect interstitial lung diseases, but the chest radiograph can be normal in up to $10 \%$ of patients, especially early on the disease process [19] Computed tomography (CT), including a specific technique known as high resolution $\mathrm{CT}$, is used to see fine detail of the interstitium that may not be visible on a chest $\mathrm{x}$-ray.

In some cases, a specific diagnosis (such as idiopathic pulmonary fibrosis) can be confirmed based on the CT appearance, potentially avoiding the need for lung biopsy. We have not included any case of biopsy or bronchoscopy.

Lungmass: Lung cancer is a leading cause of premature death. Early diagnosis is associated with improved outcomes. 
While chest $X$-ray remains the first line investigation, individual studies have indicated that a proportion of lung cancers are not identified or there are false negatives. This probably is one cause of delayed diagnosis. All the patients presenting with soft tissue mass in could not be effectively evaluated due to financial constraints as well as poor prognosis. They simply left the hospital against medical advice only 8 cases proved to have bronchogenic carcinoma, remaining 72 cases werediagnosed only radiologically.

Those which were confirmed ( 8 cases) showed signs of non-resolving consolidations and effusions. Feedback from family was not received regarding outcome of such cases.Bradley et al [20] studied 1557 patients of whom 954 (61.27\%) had a positive chest X-ray. They also suggested that nearly $25 \%$ cases of lung masses may be missed on CXR.

Pleural disease:Cases with pleural disease were not uncommon as shown in table (160 cases or $6.4 \%$ ) in present study. CXR remains mainstay in diagnosis of pleural disease. It is however non specific and less reliable for further investigation and management.

Contrast enhanced CT and ultrasound are additional investigation to establish diagnosis. In present study, The main pleural disorders were: effusion (60), thickening (80), masses(4) and pneumothorax(21)

Cardiac Cases: 102 cases with shortness of breath on exertion and other cardiac complaints were suspected to have underlying heart failure. They weresent for cardiac echo to confirm the diagnosis. $68(66.6 \%)$ patients were diagnosed to have left ventricular failure with ejection fraction less than $45 \%$ (systolic dysfunction). This study suggests that chest radiography is only moderately accurate in the diagnosis of CHF in contemporary patients presenting with acute dyspnea to the emergency department. Radiographic findings of $\mathrm{CHF}$ are specific but only moderately sensitive.

\section{Conclusion}

This study certainly has some obvious limitations. Many times, we were not able to follow the cases due to financial or other constraints. Pneumonia and other infective exacerbations are clubbed in the same group. Subgroup analyses could not be made due to logistic problem in several conditions like ILD or COPD.
Despite these limitations our study highlights the spectrum of the disease presenting to rural medical college in northern India. Though, it lacks the importance of an epidemiological survey, it still merits importance as it gives an overall idea about the prevailing chest problems in a community. It gives impetus for further in-depth analysis that may be worth while for inadequate control of certain problems in the community.

\section{Contributions of different authors}

The first author (R. Khare) was responsible for doing ultrasound and CT scanwhile technical staff carried out routine $\mathrm{X}$-ray.

Second author (A. K. Khare) was responsible for screening of CXR done and follow up of cases as well as manuscript preparation.

Declaration: We have not received any financial assistance from any agency, government or private. There is no conflict of interests.

\section{Reference}

01. Chakraborty AK. Epidemiology of tuberculosiscurrent status in India. Indian J Med Res. 2004 Oct; $120(4) 248-76$.

[Crossref]

02. Jindal SK. Emergence of chronic obstructive pulmonary disease as an epidemic in India. Indian J Med Res. 2006 Dec;124(6):619-30. [Crossref]

03. Chhabra SK, Chhabra P, Rajpal S, et al. Ambient air pollution and chronic respiratory morbidity in Delhi. Arch Environ Health. 2001 JanFeb;56(1)58-64.

[Crossref]

04. Behera D, Kashyap S. Pattern of malignancy in a north Indian hospital. J Indian Med Assoc. 1988 Feb;86(2)28-9.

[Crossref]

05. Karai GS, Nath HK, Paul G, et al. Carcinoma of the lung- A record and analysis of 100 cases. Indian J Cancer. 1967 Jun;4(2)105-13. [Crossref]

06. Dasgupta A, Bagchi A, Nag S, et al. Profile of respiratory problems in patients presenting to a referral pulmonary clinic. Lung India. 2008 Jan;25(1)4-7.

doi: $10.4103 / 0970-2113.44129$ [Crossref] 
07. Karen L Lipkind, M Ed. Division of healthcare statistics. NCHS From Vital and Health Statistics of the center for Control and prevention. Advanced data, Number 276, June 10, 1996. [Crossref]

08. Chhabra SK, Kumar R, Gupta U, et al. Prediction equations for spirometry in adults from northern India. Indian J Chest Dis Allied Sci. 2014 OctDec;56(4)221-9.

[Crossref]

09. Rajpal S, Dhingra and Aggarwal. Sputum grading as predictor of treatment outcome in pulmonary tuberculosis. Ind J Tub. 2002,49;139-41.

[Crossref]

10. Gruber $P$, Swadron $S$, DeBlieux $P$ and Nelson $B$. The acute Presentation of Chronic Obstructive pulmonary Diseases in the Emergency Department- A Challenging Oxymoron. Emergency Medicine Practice. 2008;10;11;1-27. [Crossref]

11. BTS COPD Consortium (2005). Spirometry in practice - a practical guide to using spirometry in primary care. British Thoracic Society, London. 8-9.

[Crossref]

12. Pudney $E$ and Doherty $M$. Plain chest $X$-ray (CXR) in the diagnosis of chronic obstructive pulmonary disease (COPD)". European Respiratory J. 2016;48;60.

DOI: $\quad 10.1183 / 13993003$ [Crossref]

13. Chugh, T, Goel N and Kumar. Physiological and radiological correlation in COPD. Ind $\mathrm{J}$ Chest Dis And Allied Sci. 2012;54;235-242.

[Crossref]

14. Policy Recommendations for smoking cessation and treatment of tobacco dependence. World Health Organization Tobacco Free initiative. 2008; 15-40.

[Crossref]
15. Jindal SK. Emergence of chronic obstructive pulmonary disease as an epidemic in India. Indian J Med Res. 2006 Dec;124(6)619-30.

[Crossref]

16. Horne DJ Royce, SE Gooze, L Narita, M M, Hopewell, PM Nahid, P \& Steingart, KR. "Sputum Monitoring during Tuberculosis Treatment for Predicting Outcome- A Systematic Review and Meta-analysis". Lancet Infect Dis. 2010;10(6)387-394.

[Crossref]

17. World Health organization. Definitions and reporting framework for tuberculosis- 2013. Geneva, WHO. 2016.

[Crossref]

18. van Cleeff MR, Kivihya-Ndugga LE, Meme $H$, et al. The role and performance of chest X-ray for the diagnosis of tuberculosis- a costeffectiveness analysis in Nairobi, Kenya. BMC Infect Dis. 2005 Dec 12;111.

[Crossref]

19. Attili AK, Kazerooni EA, Gross $\mathrm{BH}$, et al. Smoking-related interstitial lung disease: radiologic-clinical-pathologic correlation. Radiographics. 2008 Sep-Oct;28(5)1383-96.

doi: 10. 1148/rg.285075223 [Crossref]

20. Bradley S, Abraham S, Grice A, López PR, Wright J, Farragher T, Shinkins $B$ and Neal RD. Sensitivity of chest X-ray for lung cancersystematic review. Br J Gen Pract. 2018;68;1. [Crossref]

21. Moon JN. "Diagnostic tools of pleural effusion". Tuberc Respir Dis (Seoul). 2014 May;76(5)199210.

[Crossref] 